\title{
Sonography of the Normal and Abnormal Intact Lumbar Spinal Canal
}

\author{
Luiz A. Portela ${ }^{1}$
}

Congenital and surgical defects of the neural arches of the spine have provided sonic windows and new applications for sonography in neuroradiology $[1,2]$. The contents of the lumbar spinal canal can also be seen by an anterior approach, through the intervertebral disk, as illustrated in a recent atlas of sonography [3]. However, no previous report has been found on the use of sonography in the diagnosis of pathology of the intact lumbar spinal canal at the level of intervertebral disks.

\section{Subjects and Methods}

Since December 1981, we have endeavored to evaluate with sonography all patients referred for lumbar myelography. Clinical indications have been back pain and/or sciatica, and in all a tentative diagnosis of herniated nucleus pulposus has been advanced. Sonography was performed prospectively, minutes before myelography, with a real-time mechanical sector scanner (Combisom 100, Kretztechnik, Austria). A 2.5 MHz transducer was used routinely, placed midway between the umbilicus and pubis of the supine patient, and angled cranially. As transverse images were generated, position and angle were adjusted, sweeping up along the sacrum until the lowermost intervertebral disk space was visualized. More craniad spaces were studied in a similar manner, slowly displacing the transducer and adjusting its angle. The legs could be flexed to reduce the lumbar lordosis, and an off-center position of the transducer, aimed to the contralateral side, could enhance visualization of the lateral recess. The last two disk spaces were evaluated systematically, more craniad spaces being included when warranted clinically (fig. 1).

One hundred fifty consecutive patients are included in this report. The nature and experimental character of sonography for this indication was explained, and consent was obtained from all. The spinal canal had also been visualized in a number of patients referred for pelvic or abdominal sonography, with no symptoms referred to the spine. These studies helped us define the normal sonographic anatomy.

\section{Results}

In about one-third of the patients, an adequate penetration could not be obtained because of obesity, advanced degen- erative changes, or abnormal spinal curvatures. The normal appearance we describe is from patients with normal myelograms who became asymptomatic after clinical treatment. Bulging intervertebral disks were diagnosed using classical radiographic criteria $[4,5]$, and they were confirmed surgically in two cases. Herniated disks were diagnosed by the criteria given in the same sources, and surgical confirmation was obtained in 22 cases. Conclusions were derived only from these proven cases. No patient was explored solely on the basis of sonographic findings, although in two of the operated cases, myelography was normal, and corroboration of the sonographic findings was obtained by computed tomography (CT).

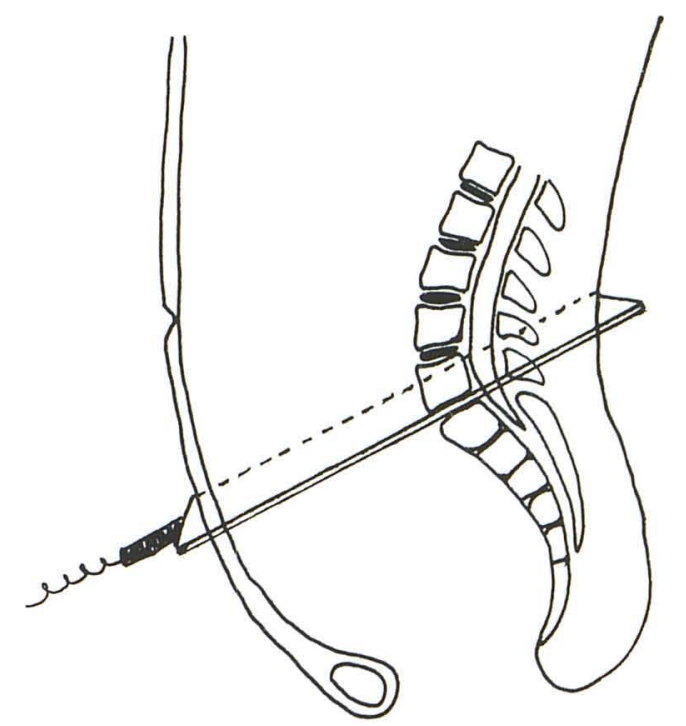

Fig. 1.-Plane of sonographic section for imaging L5-S1 disk space through anterior approach.

This article appears in the November/December 1984 issue of AJNR and in the February 1984 issue of AJR.

Received March 12, 1984; accepted April 23, 1984.

${ }_{1}^{1}$ Department of Radiology, Instituto de Neurologia de Goiania, and Clinica São Camilo, Rua 6-A 158, Setor Aeroporto, Goiania, Goias, Brazil.

AJNR 5:791-795, November/December 1984 0195-6108/84/0506-0791 \$00.00 @ American Roentgen Ray Society 

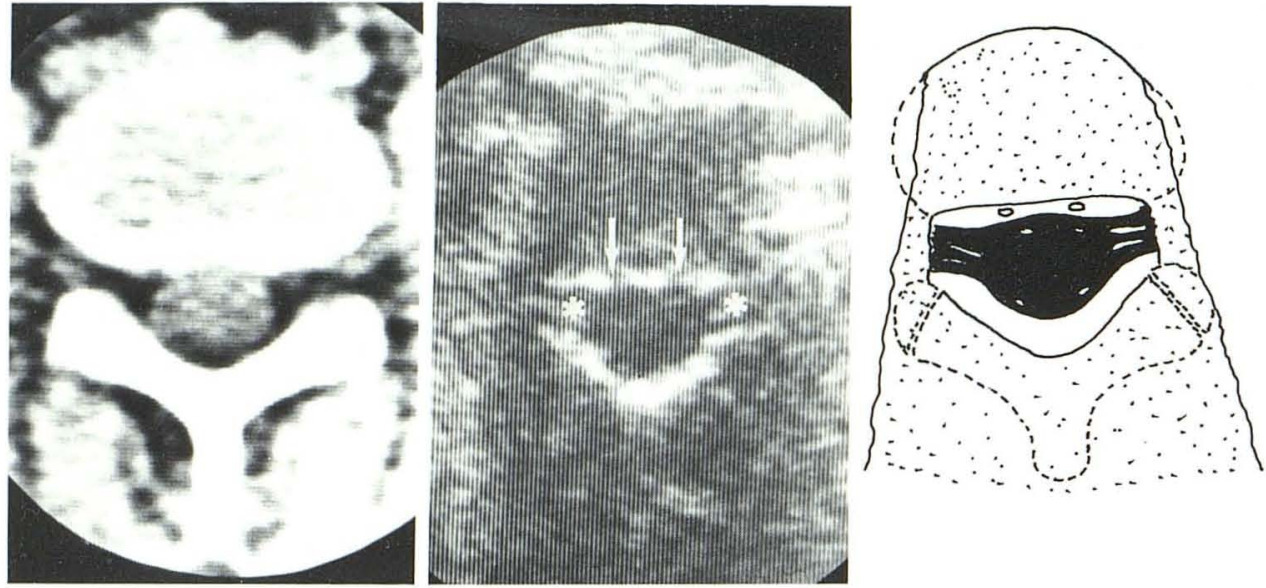

A
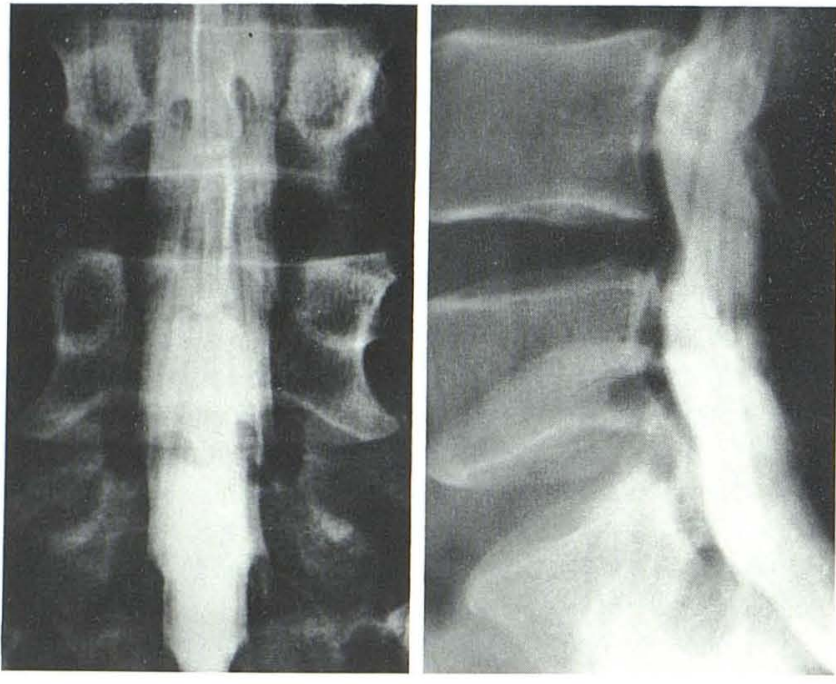

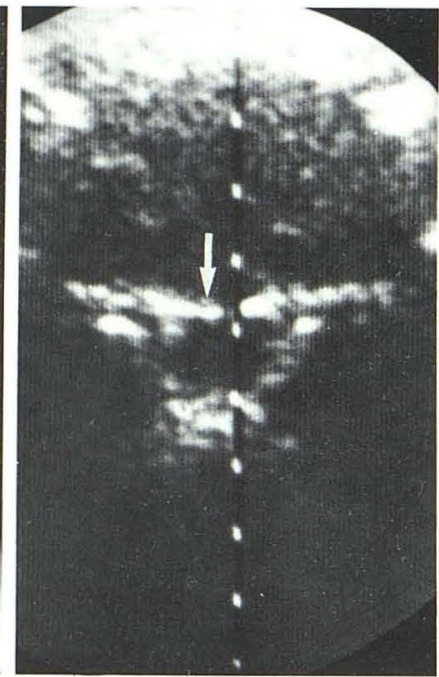

C
Fig. 2.-A, CT image at level of L4 L5 disk space. B, Sonogram at same leve in another patient. Epidural veins (arrows) and exceptionally well seen radicular sheaths (asterisks) within echogenic epidural fat. Posterior elements are not identified. C, Drawing of B with bone elements outlined. Lateral borders of disk (broken lines) are not defined.
B

A

\section{Normal Spinal Canal}

The dural sac is seen as a rounded or elliptic anechoic structure within which the roots of the cauda equina may be identified. The surrounding epidural fat is highly echogenic and interrupted by the anechoic defects caused by the epidural venous plexus. When the radicular sheaths are long or capacious they are also identified as they leave the dural sac or enter the lateral recess. The image obtained is similar to that of CT (fig. 2), except for the osseous elements posteriorly. The ligamenta flava are not identified separately from the laminae, and the lateral contour of the disk is not defined, since the sonic beam is tangential to this part and thus has no reflective surface. Care must be taken to precisely parallel the intervertebral space, since a border of the adjacent vertebrae, when partially included in the sonic beam with a partialvolume effect, will induce artifacts that may simulate a herniated disk. However, if this happens the echogenic line that is produced is more regular and follows the contour of the posterior vertebral margin.

\section{Bulging Intervertebral Disk}

The bulging disk has a convex posterior border that is not recognized easily with sonography (fig. 3 ). The substance of the disk is often less homogeneous, but this finding is dependent on the gain settings used and is quite subjective.

\section{Herniated Disk}

The herniated nucleus pulposus that remains at the level of the intervertebral space has a distinctive appearance. It is seen as a small anechoic image separated from the substance of the disk by an intensely echogenic line. The dural sac may be deformed, but only the epidural fat will be obliterated in lateral herniations (figs. 4 and 5). Compression of the radicular sheath is not identified by sonography, except in those cases in which the sheath is capacious and can be seen on the normal side. In two cases a lateral herniation recognized by sonography because of this distinctive appearance was con- 
Fig. 4.-A and B, Myelograms demonstrate herniated nucleus pulposus at L4-L5. Anterior compression of dural sac and obliteration of left $L 5$ radicular sheath (arrows). Conjoined L5 and S1 nerve roots on right (asterisk). C, Sonogram at same level. Echogenic line (long arrow) separating large herniated fragment (asterisk) from substance of disk. Dural sac displaced posteriad and left lateral recess compromised (short arrow)

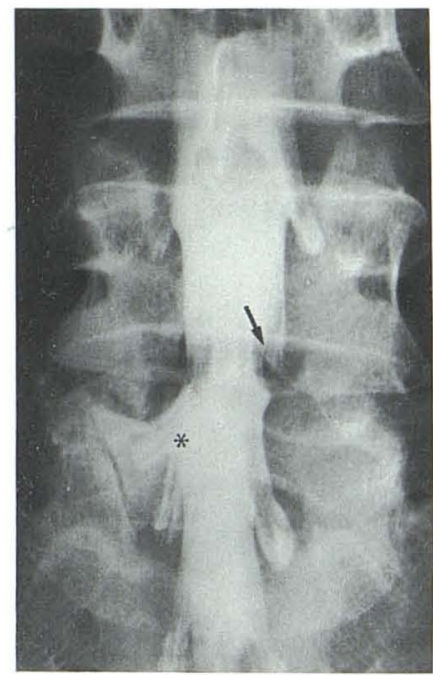

A

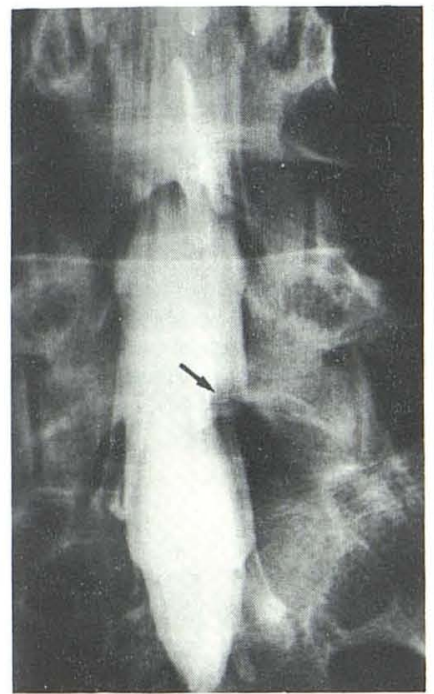

A

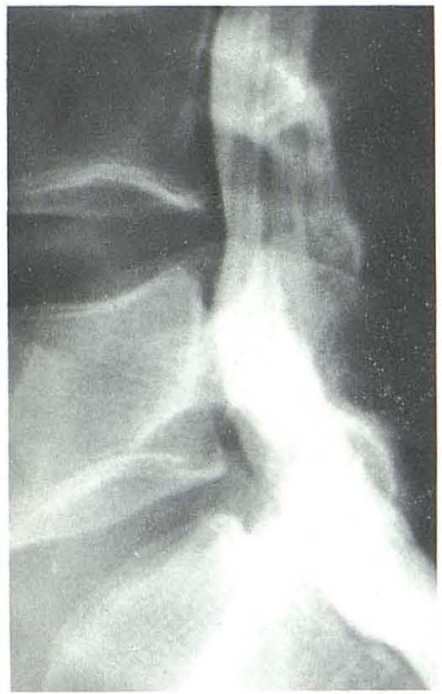

B

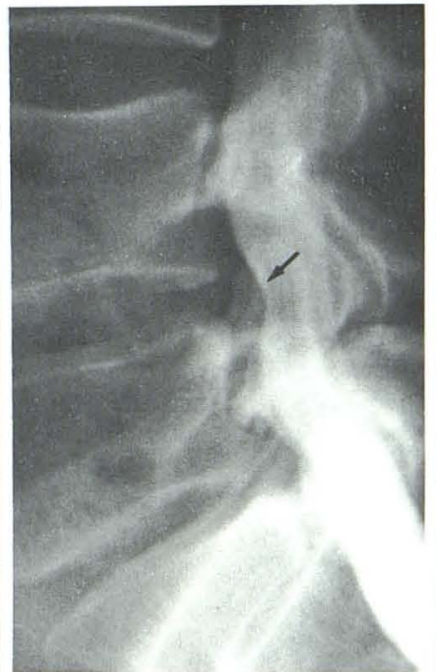

B

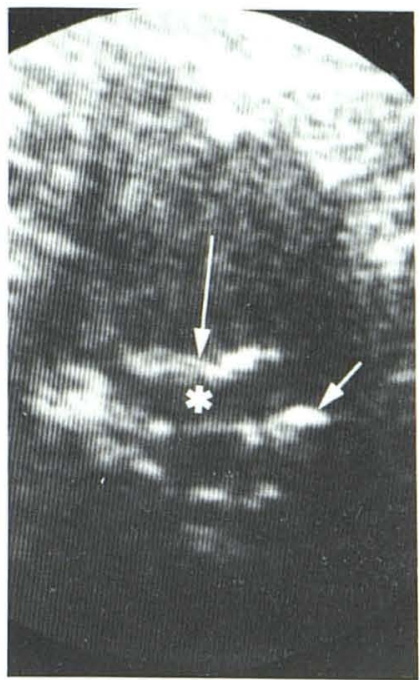

C

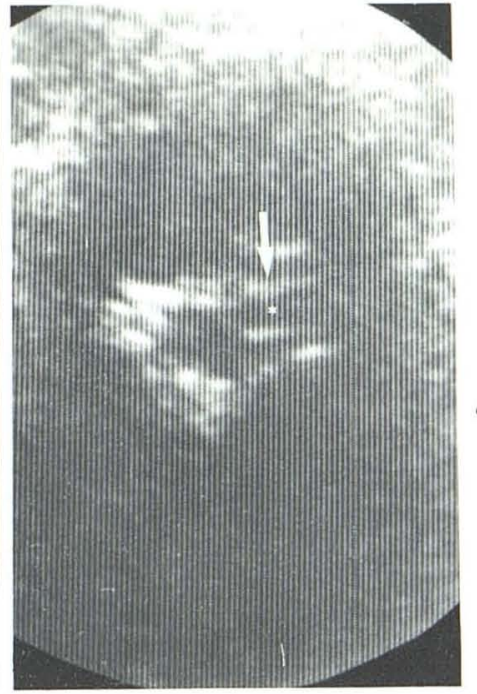

C

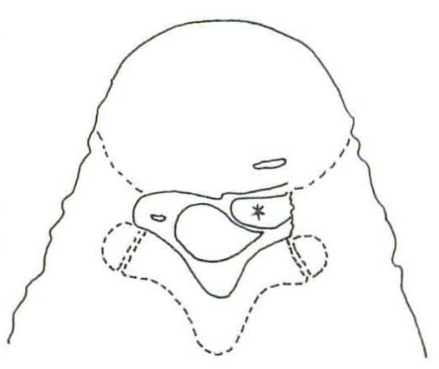

Fig. 5.-A and B, Myelograms demonstrate minimal deformity of dural sac and obliteration of left S1 radicular sheath (arrow). C, Sonogram at L5-S1 disk space. Herniated fragment (asterisk) is separated from disk by echogenic line (arrow). Epidural fat at lateral recess is obliterated.

firmed by CT and surgery, despite a normal myelogram (fig. 6).

When the herniated fragment migrates from the disk space lodging behind a vertebral body, it is not recognized by sonography. In three patients (of the 22 who had surgery), this occurred. In all three, however, the roots of the cauda equina at the disk space appeared thickened and distinctly abnormal, even though no herniated fragment could be recognized (fig. 7).

\section{Discussion}

Real-time sonography, although technically less demanding than static imaging, is more dependent on the live impression obtained during the examination. Frozen images give a poorer picture than those obtained during actual scanning. Since visualization of spinal contents is highly dependent on proper angulation of the transducer, we did not attempt to use static scanning. Sporadic use of higher-frequency transducers has suggested that their penetration is insufficient for adequate imaging of the spinal canal through this approach.

Since the sonogram of the spinal canal is analogous in plane of section to that of CT, a systematic comparison of these two methods would be valuable. In the few cases we were able to compare, an excellent correlation was found. Familiarity with $\mathrm{CT}$ images and literature has been important in our interpretation, and from studying one report [6], we speculate that the echogenic line so characteristic of the 


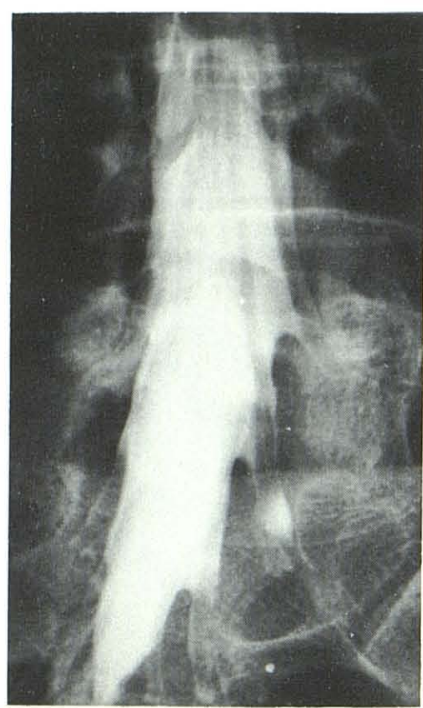

A

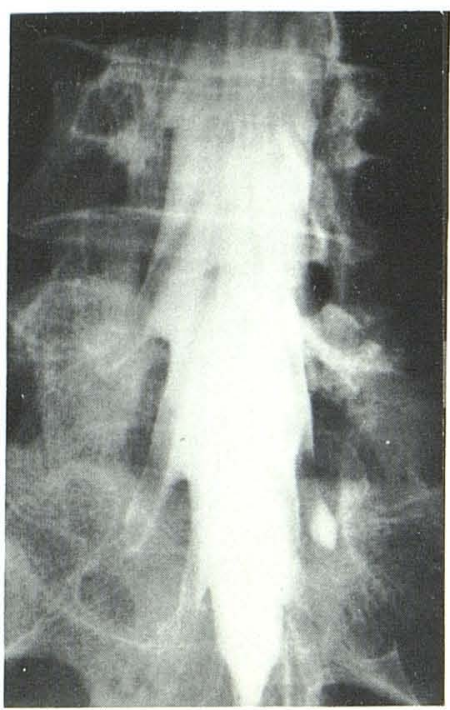

B

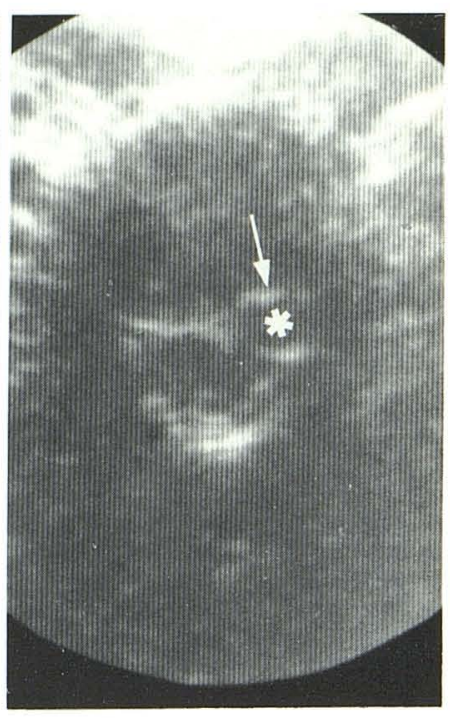

C

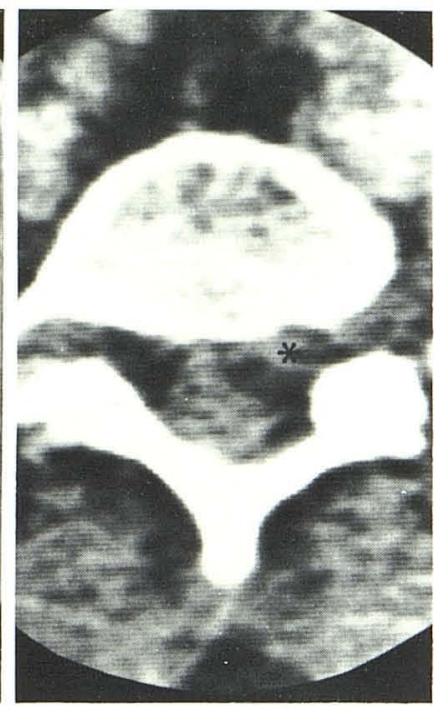

D

Fig. 6.-A and B, Myelograms are normal in patient with left sciatica. C, at L5-S1 disk space. Lateral hernia (asterisk) obliterates epidural fat of lateral recess. Echogenic line (arrow) is characteristic. D, CT corroborates finding (asterisk) confirmed by surgery.

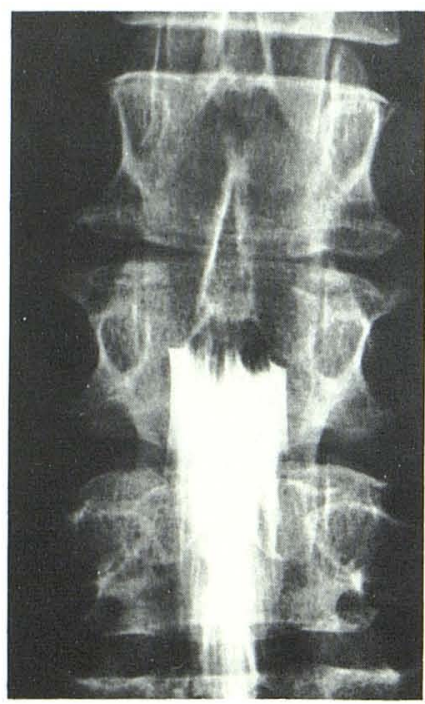

A

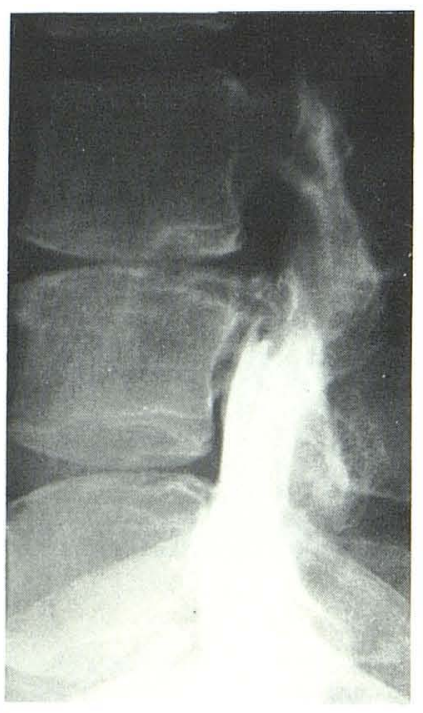

B

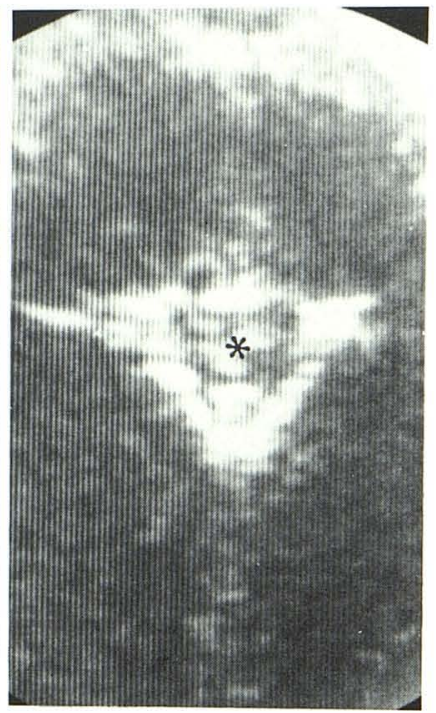

C
Fig. 7-A and B, Myelograms demonstrate complete block of subarachnoid space by extradural process slightly caudad to L3-L4 disk space. C, Sonogram at L3-L4 disk space. Thickened roots within small subarachnoid space (asterisk). Epidural fat is not obliterated and herniated fragment cannot be identified. Surgery disclosed large hernia that migrated caudad to disk space. herniated disk results from the new interface created by the rupture of the anulus fibrosus [6].

In our experience, real-time sonography, performed by a physician, can demonstrate a herniated nucleus pulposus on two conditions: (1) it is localized at the level of the intervertebral space and (2) factors such as obesity, excessive bowel gas, abnormal spinal curvature (marked scoliosis or lumbar lordosis), or advanced degenerative changes (large marginal osteophytes or vacuum or calcified disks) are not present. Correlation with plain radiographs is necessary for proper identification of the intervertebral level that is imaged, since there is no equivalent to the CT scanogram.

We have had no false-positive diagnoses of hernia. False negatives are expected to occur, although the canal was not normal (thickened roots) in the three cases in which the herniated fragment was not seen. Data from the literature show that the migrated disk fragment can be totally unsuspected at the intervertebral space [7].

With these limitations in mind, and considering that surgeons are hesitant to operate on the lumbar spine without a myelogram [8], sonography may be considered an alternative to CT in cases with a definite clinical picture of herniated nucleus pulposus in which myelography is normal or not conclusively abnormal, as a first step and to exclude a lateral hernia. Since a herniated disk is so distinctive and easily differentiated from the bulging disk, sonography may comple- 
ment CT when the latter is inconclusive.

Sonography should probably not be the primary imaging method for several reasons: (1) a normal study does not exclude significant pathology; (2) surgeons are less adept at interpreting sonograms than at interpreting other kinds of studies; (3) objective documentation of the level being studied is not assured; and (4) some patients are unsuited for sonographic study because of the technical problems discussed above.

However, sonography in conjunction with lumbar myelography may be preferable if CT scanning is not available, particularly when it is considered that some myelograms may be negative or equivocal in patients who have a herniated intervertebral disk.

The application of sonography in postoperative patients and in the diagnosis of spinal stenosis awaits further experience.

\section{ACKNOWLEDGMENTS}

I thank Michael Huckman and Claire Smith, Rush-PresbyterianSt. Luke's Medical Center, Chicago, for critical review of the original manuscript.

\section{REFERENCES}

1. Raghavendra BN, Epstein FJ, Pinto RS, Subramanyam BR, Greenberg J, Mitnick JS. The tethered spinal cord: diagnosis by high resolution real-time ultrasound. Radiology 1983;149:123128

2. Rubin JM, Dohrmann GJ. Intraoperative ultrasonography of the spine. Radiology 1983;146:173-175

3. Morley P, Donald G, Sanders R, eds. Ultrasonic sectional anatomy. London: Churchill Livingstone, 1983:54

4. Taveras JM, Wood E. Diagnostic neuroradiology, 2d ed. Baltimore: Williams \& Wilkins, 1976

5. Kieffer SA, Sherry RG, Wellenstein DE, King RB. Bulging lumbar intervertebral disk: myelographic differentiation from herniated disk with nerve root compression. AJNR 1982;3:51-58, AJR 1982;138:709-716

6. Jahnke RW. Low density in the posterior portion of the lumbar disk: a new CT finding in disk herniations. J Comput Assist Tomogr 1983;7:313-315

7. Fries JW, Abodeely DA, Vijungco JG, Yeager VL, Gaffey WR. Computed tomography of herniated and extruded nucleus pulposus. J Comput Assist Tomogr 1982;6:874-887

8. Simeone FA, Rothman $\mathrm{RH}$. Clinical usefulness of CT scanning in the diagnosis and treatment of lumbar spine disease. Radiol Clin North Am 1983;21:197-200 